05,13

\title{
Модификация поверхностного взаимодействия Дзялошинского-Мория в пленках Со/тяжелый металл при облучении ионами гелия
}

\author{
(C) Н.С. Гусев ${ }^{1}$, Ю.А. Дудин ${ }^{2}$, А.В. Садовников ${ }^{3,4}$, М.В. Сапожников ${ }^{1,5,9}$ \\ ${ }^{1}$ Институт фризики микроструктур РАН, \\ Нижний Новгород, Россия \\ ${ }^{2}$ Научно-исследовательский физико-технический институт \\ Нижегородского государственного университета им. Н.И. Лобачевского, \\ Нижний Новгород, Россия \\ 3 Лаборатория „Магнитные метаматериалы“ Саратовского государственного университета, \\ Саратов, Россия \\ ${ }^{4}$ Институт радиотехники и электроники им. В.А. Котельникова РАН, \\ Москва, Россия \\ ${ }^{5}$ Государственный университет им. Н.И. Лобачевского, \\ Нижний Новгород, Россия \\ ฯ E-mail: msap@ipmras.ru
}

Поступила в Редакцию 9 апреля 2021 г.

В окончательной редакции 9 апреля 2021 г.

Принята к публикации 19 апреля 2021 г.

Проведено экспериментальное исследование влияния облучения ионами гелия на поверхностное взаимодействие Дзялошинского-Мория (ПВДМ) в тонких пленках кобальта на слое тяжелых металлов $\mathrm{Pt}$ и W. Измерение величины ПВДМ проводилось методами мандельштам-бриллюэновской спектроскопии (МБС). Измерение петель намагничивания образцов было выполнено методами оптической магнитометрии. Обнаружено немонотонное изменение константы ПВДМ в пределах $0.1-0.6 \mathrm{~J} / \mathrm{m}^{2}$ при флюенсах $10^{14}-10^{16} \mathrm{~cm}^{-2}$.

Ключевые слова: ферромагнитные пленки, поверхностное взаимодействие Дзялошинского-Мория, перпендикулярная анизотропия.

DOI: 10.21883/FTT.2021.09.51250.04H

\section{1. Введение}

Теоретически топологические солитонные решения для распределения намагниченности известны с конца 70 -х годов $[1,2]$, но пристальное внимание к ним было привлечено лишь после обнаружения подобных состояний в хиральных магнитных материалах без центра инверсии [3-5]. Начиная с этого времени за ними закрепилось название магнитных скирмионов [6]. Недостатком хиральных магнетиков является то, что взаимодействие Дзялошинского-Мория, которое стабилизирует магнитные скирмионы обычно является в них слабым. Поэтому скирмионы могут быть устойчивы только в узкой области магнитных полей и низких температур [7]. В сверхтонких многослойных пленках из ферромагнитных и тяжелых металлов симметрия пространственной инверсии нарушается на границе между слоями, что приводит к появлению сильного поверхностного взаимодействия Дзялошинского-Мория (ПВДМ) [8], которое стабилизирует магнитные скирмионы при комнатной температуре. К настоящему времени хиральные магнитные структуры, обусловленные ПВДМ, экспериментально наблюдались в пленках $\mathrm{Mn} / \mathrm{W}$ [9], $\mathrm{Fe} / \mathrm{Ir}$ [10], Co/Pt [11]. Несмотря на то что устойчивость магнитных скирмионов может быть обусловлена ПВДМ, отдельно стоит вопрос о способах их зарождения в системе, которая изначально находится в однородном состоянии. Один из способов - пропускание тока в геометрически неоднородных мостиках [12]. Другой вариант - перемагничивание наноструктурированной магнитной пленки в однородном магнитном поле [13]. В частности такое наноструктуирование может быть обеспечено локальным облучением пленки пучком ионов гелия $[14,15]$, что само по себе может привести к стабилизации скирмиона в области с пониженной величиной магнитной анизотропии даже в отсутствие ПВДМ [16].

Хорошо известно, что коэффициент анизотропии магнитных многослойных пленок $\mathrm{Co} / \mathrm{Pt}$ может быть изменен облучением ионами Не $[17,18]$. При малых флюенcax величина перпендикулярной анизотропии уменьшается, при больших величинах пленка может приобрести анизотропию типа „легкая плоскость“. Такое изменение анизотропии обусловлено перемешиванием атомов на границе двух металлов. Величина ПВДМ обусловлена теми же самыми граничными эффектами на границе между ферромагнитным металлом и тяжелым металлом с сильным спин-орбитальным взаимодействием, как и величина перпендикулярной магнитной анизотропии. Соответственно она также должна претерпевать изменение при облучении пленок ионами гелия. Влияние 


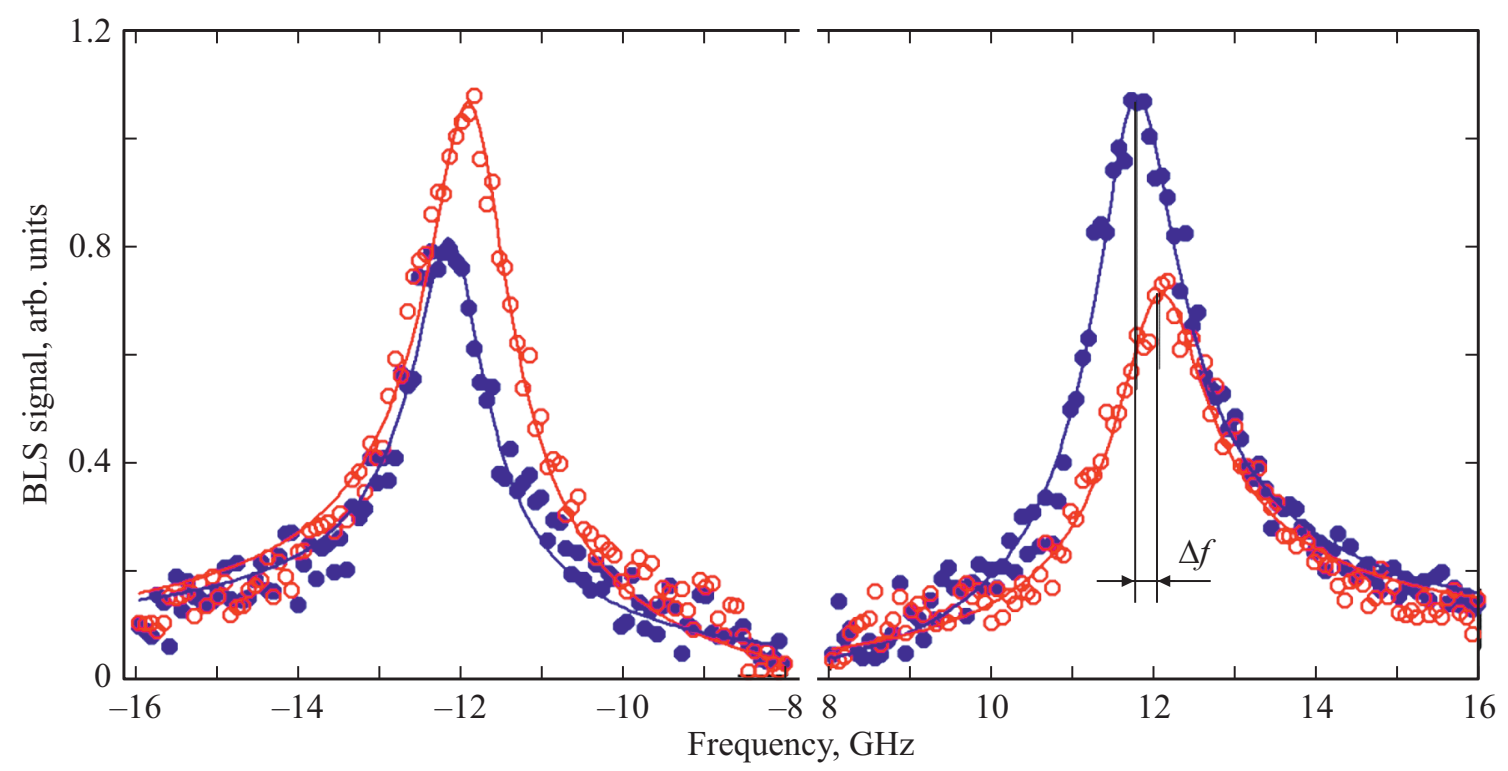

Рис. 1. Спектры МБС для пленки кобальта на поверхности тяжелого металла (приведенные данные соответствуют необлученной пленке $\mathrm{Co} / \mathrm{Pt}$ ) при $H$ равном $1 \mathrm{~T}$ (красные окружности) и $H$ равном $-1 \mathrm{~T}$ (синие кружки). Сплошные линии соответствуют лоренцевой аппроксимации экспериментальных данных. $\Delta f-$ сдвиг частоты между стоксовым и антистоксовым пиками. (Рисунок в цветном изображении см. в эл. версии журнала).

неровностей границы на величину ПВДМ было рассчитано как теоретически, на основе $a b$ initio вычислений [19], так и наблюдалось экспериментально для трехслойных структур $\mathrm{Ta} / \mathrm{CoFeB} / \mathrm{MgO}[20]$ и пленок из сплава $\mathrm{CoPt}$ [21] облученных ионами гелия. Другой альтернативный способ изменения взаимного расположения атомов на границе магнитного и тяжелого металлов это механическая деформация - сжатие или растяжение образца. Недавно было продемонстрировано, что подобные деформации приводят к значительному изменению величины ПВДМ [22].

В представленной работе нами проведено исследование влияния облучения ионами гелия на величину намагниченности, анизотропию, коэрцитивные свойства и ПВДМ в пленках Со на поверхности тяжелых металлов Pt и W, демонстрирующих сильное спин-орбитальное взаимодействие. Показано, что при величинах флюенса $10^{14}-10^{16} \mathrm{~cm}^{-2}$, происходит немонотонное изменение величины ПВДМ, в то время как величина намагниченности остается неизменной. Кроме того, пленки $\mathrm{Co} / \mathrm{Pt}$ демонстрируют хорошо известное изменение анизотропии с типа „легкая ось“ на тип „легкая плоскость“" при флюенсах $\sim 3 \cdot 10^{15} \mathrm{~cm}^{-2}$.

\section{2. Образцы и методика проведения эксперимента}

Магнитные пленки выращивались методом магнетронного напыления в атмосфере $\mathrm{Ar}$ (давление $4 \cdot 10^{-3}$ Torr) на стеклянную подложку $22 \times 22 \mathrm{~mm}^{2}$ с буферным слоем Тa $(10 \mathrm{~nm})$. Затем напылялся слой тяжелого металла $\mathrm{Pt}$ или $\mathrm{W}$ толщиной $10 \mathrm{~nm}$ и слой Со толщиной $1 \mathrm{~nm}$.
Толщина пленки Со для изготовления образцов была выбрана возможно более тонкой, так как ПВДМ возникает на поверхности, и его влияние на магнитные свойства образца уменьшается с увеличением толщины магнитной пленки. Скорость роста слоев составляет $0.125 \mathrm{~nm} / \mathrm{sec}$ для Со и $0.25 \mathrm{~nm} / \mathrm{sec}$ для Pt. Для предотвращения окисления магнитного слоя он закрывался слоем легкого металла $(\mathrm{Al})$ толщиной $5 \mathrm{~nm}$. В дальнейшем каждый образец разделялся на 8 частей размером $\sim 5 \cdot 10 \mathrm{~mm}^{2}$ и эти части подвергались однородному облучению ионами $\mathrm{He}^{+}$. Облучение проводилось на ускорителе ИЛУ-3 ионами $\mathrm{He}^{+}$с энергией $30 \mathrm{keV}$ при значениях флюенса ионов $10^{14}, 3 \cdot 10^{14}, 6 \cdot 10^{14}, 10^{15}, 3 \cdot 10^{14}$, $10^{16} \mathrm{~cm}^{-2}$. Такая методика изготовления образцов гарантирует то, что образцы с разным флюенсом будут иметь идентичную исходную структуру. Необлученная часть образца оставалась в качестве образца сравнения. Выбор энергии ионов гелия равной $30 \mathrm{keV}$ был обусловлен тем, что данный уровень энергии стандартно является максимальным уровнем энергии ионов гелия в коммерчески доступных установках литографии ФИП (фокусировнными ионными пучками) и позволяет достигать наилучшей фокусировки, а следовательно и литографического разрешения $[14,15,23]$. Глубина проникновения при этом составляет сотни микрон, соответственно имплантации ионов Не в сами тонкопленочные металлические структуры не происходит, ионы накапливаются глубоко в подложке. Ионно-лучевая установка ИЛУ-3 разработана Курчатовским институтом в 60-е годы прошлого века и представляет собой ускоритель средних по массе ионов c магнитной сепарацией. Ионный пучок ленточного типа по вертикали после выхода из магнитного поля попадает на коллекторное устройство и сканируется 

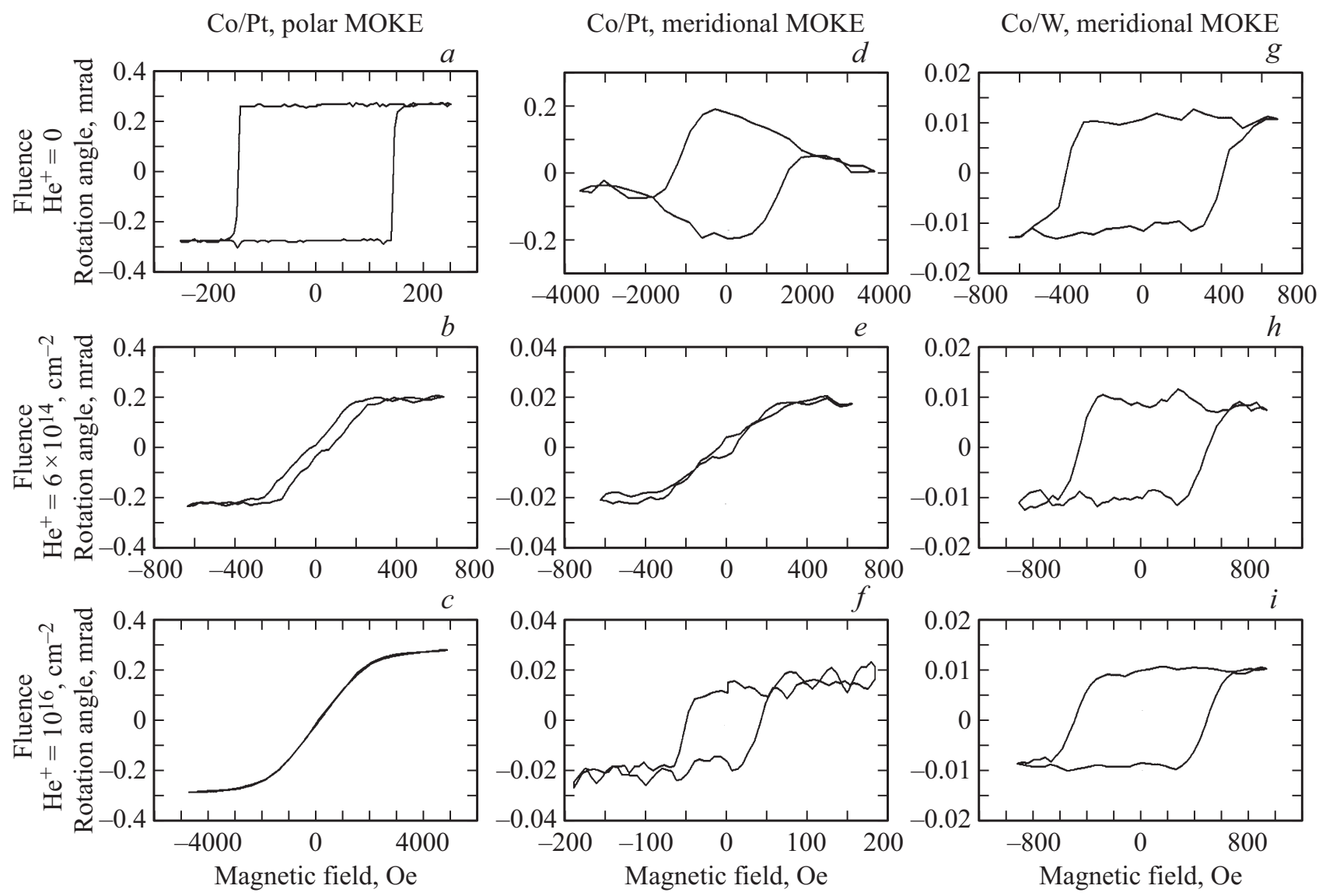

Рис. 2. Характерный вид гистерезиса МОЭК для исследуемых образцов. $a, b, c$ - полярный МОЭК в Со/Рt, $d, e, f-$ меридиональный МОЭК в $\mathrm{Co} / \mathrm{Pt}, g, h, i-$ меридиональный МОЭК в Co/W. $a, d, g$ - данные для необлученных образцов, $b, e, h-$ флюенс $6 \cdot 10^{14} \mathrm{~cm}^{-2}, c, f, i$ - флюенс $10^{16} \mathrm{~cm}^{-2}$. Видно изменение знака анизотропии пленки Со/Рt при увеличении флюенса. В том случае, когда магнитооптическое вращение измеряется в меридиональной геометрии для магнитной пленки с перпендикулярной анизотропией (необлученная пленка $\mathrm{Co} / \mathrm{Pt}$ ), вклад в измеряемый эффект дают и меридиональный и полярный эффекты.

высоким напряжением с частотой $80 \mathrm{~Hz}$ по горизонтали с амплитудой, исключающей попадание в прямоугольное отверстие в диафрагме соседних по массе ионов. Величина флюенса регулируется как плотностью потока ионов так и временем экспозиции, которое составляло по порядку десятки минут.

Исследование петель гистерезиса полученных пленок, проводилось при помощи измерения магнитооптического эффекта Керра (МОЭК) в полярной и меридиональной геометрии по методу скрещенных поляризаторов. В качестве источника излучения использовался высоко стабилизированный Не-Ne лазер $(\lambda=632 \mathrm{~nm})$. Падающий на образец свет имел $p$-поляризацию. К сожалению малая толщина пленки Со и, соответственно, слабые по величине возникающие поля рассеяния, не позволили провести измерения формирующихся в ходе перемагничивания доменных структур методами магнитносиловой микроскопии, хотя такие попытки были нами предприняты.

Для измерения величины ПВДМ был использован метод мандельштам-бриллюэновской спектроскопии (МБС) в геометрии Деймона-Эшбаха [24]. Типичный спектр BLS представлен на рис. 1. Сплошные линии показывают лоренцеву аппроксимацию, сдвиг стоксова и антистоксова пиков обозначен как $\Delta f$. Следуя стандартному подходу, константа ПВДМ оценивается как [22,24]:

$$
D=2 M_{s} \Delta f /(\pi \gamma k),
$$

где $M_{s}$ - намагниченность насыщения, $k-$ волновое число, а $\gamma=176 \mathrm{GHz} / \mathrm{T}$ - гиромагнитное отношение. Величина $M_{s}$, которую мы использовали в наших оценках, составляет $1.1 \cdot 10^{6} \mathrm{~A} / \mathrm{m}$, что является типичной величиной для тонких пленок Со $[25,26]$. Характерный вид спектра МБС приведен на рис. 1 (соответствует исходной структуре $\mathrm{Co} / \mathrm{Pt}$ )

\section{3. Экспериментальные результаты и обсуждение}

Вид петель намагничивания полученных методами магнито-оптической магнитометрии представлен на рис. 2. Исходные пленки $\mathrm{Co} / \mathrm{Pt}$ демонстрируют анизотропию типа легкая ось. Это видно по характерной петле намагничивания в перпендикулярном поле с остаточной 


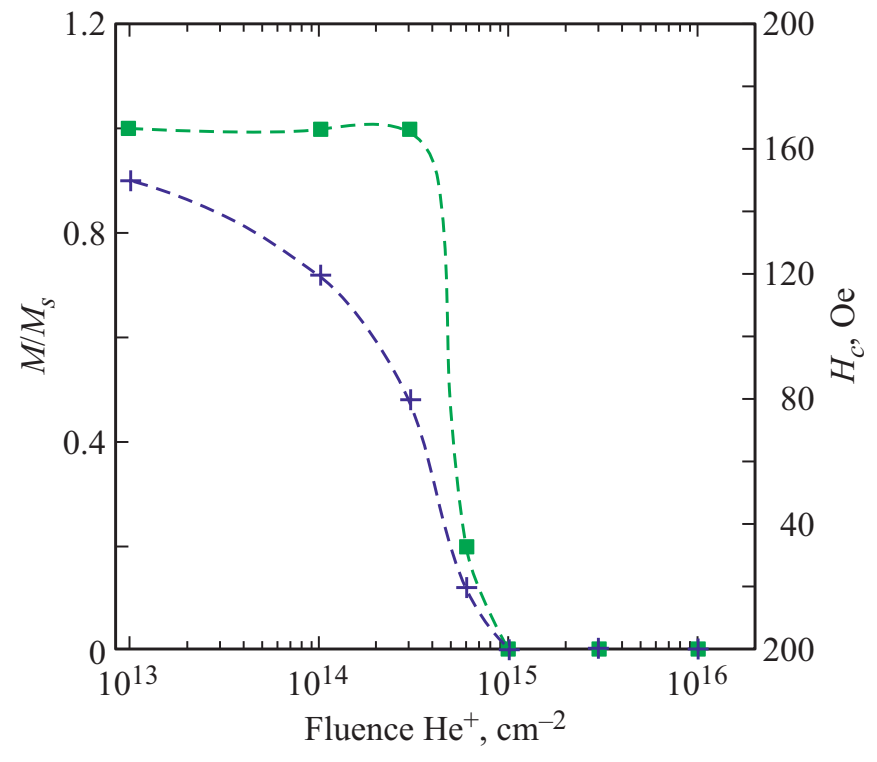

Рис. 3. Зависимость величины остаточной намагниченности (зеленые квадраты) и поля коэрцитивности (синие крестики) от флюенса ионов $\mathrm{He}^{+}$при намагничивании пленки $\mathrm{Co} / \mathrm{Pt}$ в магнитном поле перпендикулярном поверхности образца. (Рисунок в цветном изображении см. в эл. версии журнала).

намагниченностью равной намагниченности насыщения (рис. 2,a). По мере увеличения флюенса происходит постепенное уменьшение коэрцитивного поля, при этом остаточная намагниченность остается равной намагниченности насыщения вплоть до флюенса $3 \cdot 10^{14} \mathrm{~cm}^{-2}$ (рис. 3). При этом петля магнитооптического эффекта в продольном поле имеет характерный сложный вид, когда остаточный сигнал превышает сигнал в насыщении (рис. $2, d)$. Такая форма сигнала объясняется следующим. Измерения в меридиональной геометрии проводятся при угле падения света $45^{\circ}$. В этом случае волновой вектор электромагнитной волны имеет обе компоненты как компоненту, лежащую в плоскости образца, так и компоненту перпендикулярную ей. Соответственно оба эффекта, и полярный (поскольку есть остаточная намагниченность перпендикулярная поверхности) и меридиональный (поскольку намагниченность насыщения лежит в плоскости образца) дают вклад в измеряемый эффект. Соответственно наблюдаемая кривая является суммой кривой намагничивания в перпендикулярном направлении и в продольном направлении. При $6 \cdot 10^{14} \mathrm{~cm}^{-2}$ (рис. 2,b) форма кривой намагничивания изменяется, величина остаточной намагниченности $M_{z}$ уменьшается до $20 \%$, при этом остаточная намагниченность при намагничивании в плоскости (рис. $2, e$ ) становится равной 0 . Такое поведение свидетельствует о том, что хотя тип анизотропии остается „легкая ось“, ее величина уменьшается настолько, что однородно намагниченное состояние в нулевом поле становится неустойчивым и система разбивается на домены. При дальнейшем увеличении флюенса $\left(10^{15}-10^{16} \mathrm{~cm}^{-2}\right)$ остаточная $M_{z}$ становится равной 0 (рис. 2,c, рис. 3) а при намагничи- вании в плоскости появляется характерный гистерезис с остаточной намагниченностью равной намагниченности насыщения. Такое поведение явным образом свидетельствует о том, что анизотропия сменила свой тип с „легкая ось“" на „легкая плоскость“.

Наблюдаемое изменение типа анизотропии пленок $\mathrm{Co} / \mathrm{Pt}$ при облучении ионами было хорошо известно и ранее, но мы хотим обратить внимание на следующий важный экспериментальны факт. Независимо от флюенса величина магнитооптического вращения при насыщении остается неизменной и составляет $0.2 \mathrm{rad}$ в полярной геометрии и $0.02 \mathrm{rad}$ в меридиональной геометрии. Это свидетельствует о том, что в диапазоне флюенсов $10^{14}-10^{16} \mathrm{~cm}^{-2}$ величина намагниченности исследуемых пленок не меняется. Это важно в свете последующей оценки величины ПВДМ.

Что касается структур $\mathrm{Co} / \mathrm{W}$, то они изначально демонстрируют анизотропию типа легкая плоскость, при этом форма петли гистерезиса и величина остаточной намагниченности практически не зависят от флюенса $\mathrm{He}^{+}$в исследуемом диапазоне (рис. $2, g-i$ ).

Данные измерений величины ПВДМ в пленках $\mathrm{Co} / \mathrm{Pt}$ и $\mathrm{Co} / \mathrm{W}$ приведены на рис. 4. Необходимо при этом заметить, что если константа ПВДМ в Co/Pt положительна, то в $\mathrm{Co} / \mathrm{W}$ её значение отрицательно. Тем не менее, для удобства сравнения на рис. 4 представлены абсолютные значения констант в зависимости от флюенса. Величина ПВДМ оценивается по формуле (1). Полученные данные, что величина намагниченности не зависит от флюенса, позволяют сделать вывод о том, что наблюдаемое в МБС изменение сдвигов стоксового и антистоксового пиков $(\Delta f)$ связано именно с

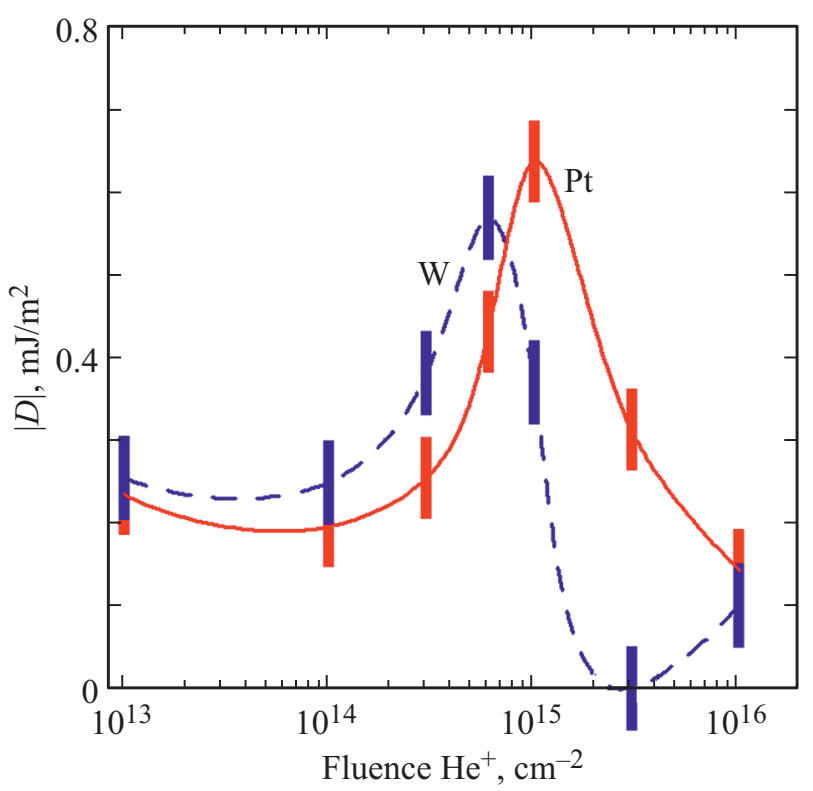

Рис. 4. Зависимость абсолютной величины коэффициента ПВДМ в пленках Со на подслое тяжелых металлов $\mathrm{Pt}$ (сплошная красная линия) и W (синяя штриховая) от флюенса ионов гелия. (Рисунок в цветном изображении см. в эл. версии журнала). 
изменением константы ПВДМ. Немонотонная зависимость $D$ от флюенса в $\mathrm{Co} / \mathrm{Pt}$ демонстрирует максимум при флюенсах $10^{15} \mathrm{~cm}^{-2}$, при этом константа ВДМ увеличивается в 3 раза по сравнению с необлученными пленками. Дальнейшее увеличение флюенса приводит к быстрому уменьшению ПВДМ. Подобный же эффект наблюдается для пленок $\mathrm{Co} / \mathrm{W}$ с той лишь разницей, что пик величины ПВДМ соответствует чуть меньшим флюенсам $-6 \cdot 10^{14} \mathrm{~cm}^{-2}$.

Наблюдаемое изменение величины ПВДМ, повидимому, связано с изменением структуры межпленочного интерфейса, но данный вопрос требует дополнительного изучения. Отметим, что о подобном немонотонном изменении константы ПВДМ в структуре $\mathrm{Ta} / \mathrm{CoFeB} / \mathrm{MgO}$ при облучении ионами сообщалось в работе [20]. При этом максимальное значение ПВДМ соответствовало флюенсу $1.2 \cdot 10^{15} \mathrm{~cm}^{-2}$, что вполне согласуется с нашими результатами. При этом в максимуме ПВДМ возрастал в 3 раза по сравнению с необлученными контрольными образцами, что так же количественно соответствует нашим результатам.

\section{4. Заключение}

В работе продемонстрирована возможность модифицирования величины взаимодействия ДзялошинскогоМории в пленках Со на поверхности тяжелого металла $(\mathrm{Pt}$ и $\mathrm{W})$ при облучении ионами гелия с энергией $30 \mathrm{keV}$. Обнаружено, что при флюенсах $\sim 6 \cdot 10^{14}-10^{15} \mathrm{~cm}^{-2}$ происходит увеличение константы ПВДМ, что должно приводить к понижению энергию доменной стенки и облегчать формирование магнитных скирмионов. Локальное модифицирование магнитных пленок ионными пучками может служить инструментом для формирования магнитных наноструктур для скирмионной спинтроники.

\section{Финансирование работы}

Работа выполнена в рамках государственного задания № 0030-2021-0021.

\section{Конфликт интересов}

Авторы заявляют, что у них нет конфликта интересов.

\section{Список литературы}

[1] И.Е. Дзялошинский, Б.А. Иванов. Письма в ЖЭТФ 29, 592 (1979).

[2] А.С. Ковалев, А.М. Косевич, К.В. Маслов. Письма в ЖЭТФ 30, 321 (1979).

[3] S. Muhlbauer, B. Binz, F. Jonietz, C. Pfleiderer, A. Rosch, A. Neubauer, R. Georgii, P. Boni. Science 323, 915 (2009).

[4] X.Z. Yu, Y. Onose, N. Kanazawa, J.H. Park, J.H. Han, Y. Matsui, N. Nagaosa, Y. Tokura. Nature (London) 465, 901 (2010).
[5] S. Heinze, K. von Bergmann, M. Menze, J. Brede, A. Kubetzka, R. Wiesendanger, G. Bihlmayer, S. Blugel. Nature Phys. 7, 713 (2011).

[6] U.K. R'ossler, A.N. Bogdanov, C. Pfleiderer. Nature 442, 797 (2006).

[7] N. Nagaosa, Y. Tokura. Nature Nanotechnol. 8, 899 (2013).

[8] A. Fert. Mater. Sci. Forum 59, 439 (1990).

[9] M. Bode, M. Heide, M.K. von Bergmann, M.P. Ferriani, M.S. Heinze, M.G. Bihlmayer, M.A. Kubetzka, M.O. Pietzsch, M.S. Blügel, M.R. Wiesendanger. Nature (London) 447, 190 (2007).

[10] S. Heinze, K. von Bergmann, M. Menzel, J. Brede, A. Kubetzka, R. Wiesendanger, G. Bihlmayer, S. Blügel. Nature Phys. 7, 713 (2011).

[11] S.-G. Je, D.-H. Kim, S.-C. Yoo, B.-C. Min, K.-J. Lee, S.B. Choe. Phys. Rev. B 88, 214401 (2013).

[12] W. Jiang, P. Upadhyaya, W. Zhang, G. Yu, M.B. Jungfleisch, F.Y. Fradin, J.E. Pearson, Y. Tserkovnyak, K.L. Wang, O. Heinonen, S.G.E. te Velthuis, A. Hoffmann. Science 349, 283 (2015).

[13] L. Sun, R.X. Cao, B.F. Miao, Z. Feng, B. You, D. Wu, W. Zhang, A. Hu, H.F. Ding. Phys. Rev. Lett. 110, 167201 (2013).

[14] M.V. Sapozhnikov, S.N. Vdovichev, O.L. Ermolaeva, N.S. Gusev, A.A. Fraerman, S.A. Gusev, Yu.V. Petrov. Appl. Phys. Lett. 109, 042406 (2016).

[15] M.V. Sapozhnikov, N.S. Gusev, S.A. Gusev, D.A. Tatarskiy, Yu.V. Petrov, A.G. Temiryazev, A.A. Fraerman. Phys. Rev. B 103, 054429 (2021)

[16] M.V. Sapozhnikov. J. Magn. Magn. Mater. 396, 338 (2015).

[17] C. Chappert, H. Bernas, J. Ferre, V. Kottler, J.-P. Jamet, Y. Chen, E. Cambril, T. Devolder, F. Rousseaux, V. Mathet, H. Launois. Science 280, 1919 (1998).

[18] T. Devolder, J. Ferre, C. Chappert, H. Bernas, J.-P. Jamet, V. Mathet. Phys. Rev. B 64, 064415 (2001).

[19] B. Zimmermann, W. Legrand, D. Maccariello, N. Reyren, V. Cros, S. Blügel, A. Fert. Appl. Phys. Lett. 113, 232403 (2018).

[20] L.H. Diez, M. Voto, A. Casiraghi, M. Belmeguenai, Y. Roussigné, G. Durin, A. Lamperti, R. Mantovan, V. Sluka, V. Jeudy, Y.T. Liu, A. Stashkevich, S.M. Chérif, J. Langer, B. Ocker, L. Lopez-Diaz, D. Ravelosona. Phys. Rev. B 99, 054431 (2019).

[21] И.Л. Калентьева, О.В. Вихрова, Ю.А. Данилов, А.В. Здоровейщев, М.В. Дорохин, Ю.А. Дудин, А.В. Кудрин, М.П. Темирязева, А.Г. Темирязев, С.А. Никитов, А.В. Садовников. ФTT 63, 324 (2021).

[22] N.S. Gusev, A.V. Sadovnikov, S.A. Nikitov, M.V. Sapozhnikov, O.G. Udalov. Phys. Rev. Lett. 124, 157202 (2020).

[23] G. Nanda, G. Hlawacek, S. Goswami, K. Watanabe, T. Taniguchi, P.F.A. Alkemad. Carbon 119, 419 (2017).

[24] K. Di, V.L. Zhang, H.S. Lim, S.C. Ng, M.H. Kuok, J. Yu, J. Yoon, X. Qiu, H. Yang. Phys. Rev. Lett. 114, 047201 (2015).

[25] L. Sun, R.X. Cao, B.F. Miao, Z. Feng, B. You, D. Wu, W. Zhang, A. Hu, H.F. Ding. Phys. Rev. Lett. 110, 167201 (2013).

[26] C. Eyrich, W. Huttema, M. Arora, E. Montoya, F. Rashidi, C. Burrowes, B. Kardasz, E. Girt, B. Heinrich, O.N. Mryasov, M. From, O. Karis. J. Appl. Phys. 111, 07C919 (2012).

Редактор Д.В. Жуманов 